\title{
A Third Route to the Doomsday Argument
}

\author{
preprint \\ Paul Franceschi \\ University of Corsica \\ revised September 2004 \\ p.franceschi@univ-corse.fr \\ http://www.univ-corse.fr/ franceschi
}

\begin{abstract}
In this paper, I present a solution to the Doomsday argument based on a third type of solution, by contrast to on the one hand, the Carter-Leslie view and on the other hand, the Eckhardt et al. analysis. I begin by strengthening both competing models by highlighting some variations of their ancestors models, which renders them less vulnerable to some objections. I describe then a third line of solution, which incorporates insights from both Leslie and Eckhardt's models and fits more adequately with the human situation corresponding to DA. I argue then that this two-sided analogy casts new light on the reference class problem. This leads finally to a novel formulation of the argument that could well be more consensual than the original one.
\end{abstract}

In this paper, I present a solution to the Doomsday argument (DA, for short) based on a third type of solution, by contrast to on the one hand, the Carter-Leslie view and on the other hand, the Eckhardt et al. analysis. In section 1, I describe the Carter-Leslie view. In section 2, I review the Eckhardt et al. line of reasoning. I point out then in section 3 an atemporal-temporal disanalogy in the Carter-Leslie analogy, which leads to the description of a strengthened variation of this latter model. In section 4, I raise some criticisms against the Eckhardt et al. analogy, thus leading to reformulate this latter analogy more accurately. I present then in section 5 a two-sided analogy that incorporates insights from both Carter-Leslie's and Eckhardt et al.'s models. Finally, I show in section 6 that this two-sided model is capable of handling the reference class problem and leads to a novel formulation of the argument. ${ }^{1}$

\section{The Carter-Leslie View}

Let us begin by sketching briefly the Doomsday argument. Begin with the two following competing hypotheses:

$\left(\mathrm{H} 1_{\text {few }}\right)$ the urn contains 10 balls

( $\left.\mathrm{H} 2_{\text {many }}\right)$ the urn contains 1000 balls

and the corresponding prior probabilities: $\mathrm{P}(\mathrm{H} 1)=\mathrm{P}(\mathrm{H} 2)=0.5$. Now the argument can be described as a reasoning leading to a Bayesian shift, from an analogy between what has been termed the two-urn case $^{2}$ and the corresponding human situation. Consider, first, the two-urn case (slightly adapted from Bostrom 1997): ${ }^{3}$

\footnotetext{
${ }^{1}$ The present account of DA finds its origin in Franceschi (2002).

${ }^{2}$ Cf. Korb \& Oliver (1998).

${ }^{3}$ Cf. Bostrom (1997): 'Imagine that two big urns are put in front of you, and you know that one of them contains ten balls and the other a million, but you are ignorant as to which is which. You know the balls in each urn are numbered 1, 2, 3, 4 ... etc. Now you take a ball at random from the left urn, and it is number 7. Clearly, this is a strong indication that that urn contains only ten balls. If originally the odds were fifty-fifty, a swift application of Bayes' theorem gives you the posterior probability that the left urn is the one with only ten balls. (Pposterior $(\mathrm{L}=10)=0.999990)^{\prime}$.
} 
The two-urn case An urn ${ }^{4}$ is in front of you, and you know that it contains, depending on the flipping at time $\mathrm{T}_{0}$ of a fair coin, either 10 (tails) or 1000 (heads) numbered balls. The balls are numbered $1,2,3, \ldots$ At this step, you formulate the $\mathrm{H}_{\text {few }}$ and $\mathrm{H}_{\text {many }}$ assumptions with $\mathrm{P}\left(\mathrm{H}_{\text {few }}\right)=$ $\mathrm{P}\left(\mathrm{H}_{\text {many }}\right)=0.5$ and you try to evaluate the number of balls which were contained at $\mathrm{T}_{0}$ in the urn. You know all the above and you randomly draw a ball from the urn at time $\mathrm{T}_{1}$. Now you get the ball \#5 at $\mathrm{T}_{1}$. You conclude then to an upward Bayesian shift in favour of the $\mathrm{H}_{\mathrm{few}}$ hypothesis.

The two-urn case constitutes an uncontroversial application of Bayes' theorem. Taking into account the fact that $E$ denotes the available evidence that the random ball is $\# 5$ and $\mathrm{P}(\mathrm{E} \mid \mathrm{H} 1)=1 / 10$ and $\mathrm{P}(\mathrm{E} \mid \mathrm{H} 2)=1 / 1000$, a Bayesian shift ensues from a straightforward application of Bayes' theorem. As a result, the posterior probability is such that $\mathrm{P}^{\prime}(\mathrm{H} 1)=0.99$.

Let us consider, on the other hand, the human situation corresponding to DA. Now being concerned with the final size of the human race, you consider the two following competing hypotheses:

$\left(\mathrm{H}_{\text {few }}\right)$ the number of humans having ever lived will reach $10^{11}$ (doom soon)

$\left(\mathrm{H} 4_{\text {many }}\right)$ the number of humans having ever lived will reach $10^{14}$ (doom later)

Now it appears that each human has his own birth rank, and that yours is roughly $60 \times 10^{9}$. Let us assume then, for the sake of simplicity, that the prior probabilities are such that $\mathrm{P}(\mathrm{H} 3)=\mathrm{P}(\mathrm{H} 4)=0.5$. $^{5}$ Now according to Carter and Leslie, the human situation corresponding to DA is analogous to the twourn case. ${ }^{6}$ Let us denote by $\mathrm{E}$ the fact that your birth rank is $60 \times 10^{9}$. Thus, an application of Bayes' theorem, taking into account the fact that $\mathrm{P}(\mathrm{E} \mid \mathrm{H} 3)=1 / 10^{11}$ and $\mathrm{P}(\mathrm{E} \mid \mathrm{H} 4)=1 / 10^{14}$, leads to a vigorous Bayesian shift in favour of the hypothesis that Doom will occur soon: $\mathrm{P}^{\prime}(\mathrm{H} 3)=0.999$. For this reason, the Carter-Leslie line of thought can be summarized as follows:

(5) in the two-urn case, a Bayesian shift of the prior probability of $\mathrm{H}_{\text {few }}$ ensues

(6) the situation corresponding to DA is analogous to the two-urn case

(7) $\therefore$ in the situation corresponding to DA, a Bayesian shift of the prior probability of $\mathrm{H}_{\text {few }}$ ensues

From the Carter-Leslie's viewpoint, the analogy with the urn is well-grounded. And it legitimates DA's conclusion according to which a Bayesian shift in favour of doom soon ensues. This last conclusion appears paradoxical or at least counter-intuitive. But the task of diagnosing what is wrong, if any, with the Doomsday Argument proves to be very difficult and remains an open question.

At this point, it is worth mentioning in passing that the reasoning based on the two-urn case does not yield absolute certainty. This last reasoning is probabilistic and as such, it leads to a true conclusion in most cases. If the experiment is repeated many times and you bet accordingly, you will win in most cases. But it must be acknowledged that you will sometimes lose. For consider the exceptional situation where the coin lands heads and the number of balls in the urn is 1000 and you get the ball \#5. In this last case, the reasoning based on the two-urn case leads to the false conclusion that the urn contains only 10 balls. However, this does not preclude us from regarding the corresponding reasoning as sound. For in the long run, it is reliable and yields many more true conclusion than false ones. The following table summarises this situation:

\begin{tabular}{cccrr}
\hline \multicolumn{5}{c}{ two-urn case (numbered balls) } \\
\hline toss outcome & reference class (numbered balls) & $\#$ & prediction & reasoning \\
\hline tail (doom soon) & 10 numbered balls & $\# 5$ & true & sound \\
\hline heads (doom later) & 1000 numbered balls & $\# 5$ & false & sound \\
\hline
\end{tabular}

\footnotetext{
${ }^{4}$ Bostrom's original description of the two-urn case refers to two urns. For the sake of simplicity, I refer here equivalently to one single urn (containing either 10 or 1000 balls).

${ }^{5}$ The reasoning remains unaltered if we consider some alternative prior probabilities (with $0<\mathrm{P}(\mathrm{H} 3)<1$ ).

${ }^{6}$ More precisely, Leslie considers an analogy with the lottery case.
} 


\title{
2. The Eckhardt et al. Analysis
}

A line of objection to the Doomsday argument initially raised by William Eckhardt $(1993,1997)$ and recently echoed by George Sowers (2002) and Elliott Sober (2003) runs as follows. The analogy with the urn at the origin of DA, so the objection goes, is ill-grounded. For in the two-urn case, the ball number is randomly chosen. But in the human situation corresponding to DA, our birth rank is not randomly chosen, but rather indexed on the corresponding temporal position. Hence, the analogy is illgrounded and the whole reasoning is invalid. Eckhardt notably stresses the fact that it is impossible to make a random selection when there exists numerous unborn members in the chosen reference class. ${ }^{7}$ Sober (2003) argues along the same lines, ${ }^{8}$ by pointing out that no mechanism having the effect of randomly assigning a temporal location to human beings, can be exhibited. Lastly, such a line of objection has been recently revived by Sowers. He emphasises that the birth rank of each human is not random, because it is indexed on the corresponding temporal position. ${ }^{9}$

In parallel, according to the Eckhardt et al. analysis, the human situation corresponding to DA is not analogous to the two-urn case, but rather to an alternative model, the consecutive token dispenser. The consecutive token dispenser is a device, initially described by Eckhardt, ${ }^{10}$ that expels consecutively numbered balls at a constant rate: '(...) suppose on each trial the consecutive token dispenser expels either 50 (early doom) or 100 (late doom) consecutively numbered tokens at the rate of one per minute'. A similar device - call it the numbered ball dispenser - is also mentioned by Sowers: ${ }^{11}$

\begin{abstract}
There are two urns populated with balls as before, but now the balls are not numbered. Suppose you obtain your sample with the following procedure. You are equipped with a stopwatch and a marker. You first choose one of the urns as your subject. It doesn't matter which urn is chosen. You start the stopwatch. Each minute you reach into the urn and withdraw a ball. The first ball withdrawn you mark with the number one and set aside. The second ball you mark with the number two. In general, the $n^{\text {th }}$ ball withdrawn you mark with the number $n$. After an arbitrary amount of time has elapsed, you stop the watch and the experiment. In parallel with the original scenario, suppose the last ball withdrawn is marked with a seven. Will there be a probability shift? An examination of the relative likelihoods reveals no.
\end{abstract}

Thus, according to the Eckhardt et al. line of thought, the human situation corresponding to DA is not analogous to the two-urn case, but rather to the numbered ball dispenser. And within this latter model, the conditional probabilities are such that $\mathrm{P}(\mathrm{E} \mid \mathrm{H} 1)=\mathrm{P}(\mathrm{E} \mid \mathrm{H} 2)=1$. As a consequence, the prior probabilities of the two alternative hypotheses $\mathrm{H}_{\text {few }}$ and $\mathrm{H}_{\text {many }}$ are unchanged. Hence, the corresponding line of reasoning goes as follows:

(8) in the numbered ball dispenser, the prior probabilities remain unchanged

(9) the situation corresponding to DA is analogous to the numbered ball dispenser

(10) $\therefore$ in the situation corresponding to DA, the prior probabilities remain unchanged

thus yielding $\mathrm{P}\left(\mathrm{H}_{\text {few }}\right)=\mathrm{P}^{\prime}\left(\mathrm{H}_{\text {few }}\right)$ and $\mathrm{P}\left(\mathrm{H}_{\text {many }}\right)=\mathrm{P}^{\prime}\left(\mathrm{H}_{\text {many }}\right)$.

\footnotetext{
${ }^{7}$ Cf. (1997, p. 256): 'How is it possible in the selection of a random rank to give the appropriate weight to unborn members of the population?'.

${ }^{8}$ Cf. (2003, p. 9): 'But who or what has the propensity to randomly assign me a temporal location in the duration of the human race? There is no such mechanism.'. But Sober is mainly concerned with providing empirical evidence against the hypotheses used in the original version of DA and with broadening the scope of the argument by determining the conditions of its application to concrete situations.

${ }^{9}$ Cf. (2002, p. 40): 'My claim is that by assigning a rank to each person based on birth order, a time correlation is established (...).' and also (2002, p. 44): 'The doomsday argument has been shown to be fallacious due to the incorrect assumption that you are a random sample from the set of all humans ever to have existed.'

${ }^{10}$ Cf. (1997, p. 251).

${ }^{11}$ Cf. (2002, p. 39).
} 


\section{Strengthening the Carter-Leslie Analogy}

As we have seen, according to the Carter-Leslie view, DA is based on an analogy between the human situation corresponding to DA and the two-urn case. By contrast, from the Eckhardt et al. standpoint, the analogy associates the human situation corresponding to DA and the numbered ball dispenser. In what follows, I shall argue that both analogies suffer from some defects and consequently do not prove fully adequate. This leads finally to reformulating the analogy more accurately.

Consider, to begin with, the analogy with the two-urn case inherent to the Carter-Leslie view. Let us consider the characteristics of the human situation corresponding to DA. A summary analysis reveals indeed that this last situation is temporal. In effect, the birth ranks are successively attributed to human beings in function of the temporal position corresponding to their appearance on Earth. Thus, the corresponding situation takes place, say, from $\mathrm{T}_{1}$ to $\mathrm{T}_{\mathrm{n}}, 1$ and $n$ being respectively the rank numbers of the first and of the last human. By contrast, the two-urn case is atemporal, for at the moment where the ball is randomly drawn, all balls are already present in the urn. ${ }^{12}$ Consequently, the two-urn case takes place at a given time $\mathrm{T}_{0}$. At this step, it is patent that the two-urn case is rendered in an atemporal model while the situation corresponding to DA needs to be modelled in a temporal one. In short, the situation corresponding to DA being temporal, and the two-urn case being atemporal precludes us from regarding the two situations as isomorphic. ${ }^{13}$ The importance of the atemporal-temporal disanalogy will become clearer later. Roughly, its importance rests on the fact that the two competing models, namely the two-urn case and the numbered ball dispenser, are not on a par, since the two-urn case is atemporal, while on the other hand, the numbered ball dispenser has a fully temporal distinctive feature. This precludes us from drawing accurate comparisons between the two models. In any case, at this step, it is apparent that the human situation corresponding to DA being temporal should be put in analogy more accurately with a temporal experiment.

The atemporal-temporal disanalogy being stated, let us investigate now how this inconvenient could be overcome. Consider then the following experiment, which can be termed the incremental two-urn case (let us denote it by two-urn case ${ }^{++}$):

The synchronic two-urn case ${ }^{++}$An urn is in front of you, and you know that it contains, depending on the flipping at time $\mathrm{T}_{0}$ of a fair coin, either 10 (tails) or 1000 (heads) numbered balls. At time $T_{1}$, you randomly draw the ball \#e from the urn. Then a device expels at $T_{1}$ the ball \#1, at $\mathrm{T}_{2}$ the ball \#2..., and finally at $\mathrm{T}_{\mathrm{e}}$ the ball \#e. ${ }^{14}$ Once the ball \#e expelled, the device stops. You formulate the $\mathrm{H}_{\text {few }}$ and $\mathrm{H}_{\text {many }}$ assumptions with $\mathrm{P}\left(\mathrm{H}_{\text {few }}\right)=\mathrm{P}\left(\mathrm{H}_{\text {many }}\right)=0.5$ and you try to evaluate the number of balls which were contained at $\mathrm{T}_{0}$ in the urn. You know all the above and you get the ball \#5 at $\mathrm{T}_{5}$ when the device stops. You conclude then to an upward Bayesian shift in favour of the $\mathrm{H}_{\text {few }}$ hypothesis.

The novelty in this variation is that the experiment presents a temporal feature, given that the random selection is made at $T_{1}$ and the chosen ball is ultimately expelled, say, at $T_{5}$. It is also worth pointing out that in the synchronic two-urn case ${ }^{++}$, the total number of balls in the urn is definitively fixed at $\mathrm{T}_{0}$, when the experiment begins. An instance of the synchronic two-urn case $e^{++}$is as follows:

\begin{tabular}{ccccccc}
\hline time & $\mathrm{T}_{0}$ & $\mathrm{~T}_{1}$ & $\mathrm{~T}_{2}$ & $\mathrm{~T}_{3}$ & $\mathrm{~T}_{4}$ & $\mathrm{~T}_{5}$ \\
\hline flipping & tails & & & & & \\
\hline range & \multicolumn{7}{c}{$1-10$} & & & & \\
\hline random \# & 5 & & & & \\
\hline expelled balls \# & 1 & 2 & 3 & 4 & 5 \\
\hline
\end{tabular}

\footnotetext{
${ }^{12}$ It could be pointed out that a small amount of time is necessary to perform the Bayesian shift, once the problem's data are known. But this can be avoided if one considers ideal thinkers, who perform Bayesian shifts at the time when they are informed of the data relevant to the corresponding situation.

${ }_{13}^{13}$ I borrow this terminology from Chambers (2001).

${ }^{14}$ From now on, I assume that the intervals of time, i. e. from $T_{1}$ to $T_{n}$, are regular. Considering alternatively irregular intervals of time would not result in significant differences in the present account.
} 
At this step, it should be emphasised that anyone who accepts the conclusion of the two-urn case would also be committed to accepting the Bayesian shift resulting from the incremental two-urn case.

Furthermore, it appears that other variations of the incremental two-urn case can even be envisaged. In particular, variations of the experiment where the random process is performed gradually instead of at a given time (i.e. at time $\mathrm{T}_{0}$ ) can be envisaged. It suffices to replace the diachronic random process of the preceding experiment with a diachronic one. Such diachronic random drawing of the ball \#e is performed as follows: at $\mathrm{T}_{\mathrm{i}}$, the robot draws a ball at random in the urn (now containing the balls \#i to $\# n)$ and the device expels the ball $\# i$; if the ball $\# i$ has been drawn at $T_{i}$ then the device stops at $T_{i}$. To give an example. At time $\mathrm{T}_{1}$, the robot draws the ball \#4 at random in the urn (containing the balls \#1 to \#n), replaces it in the urn and then expels the ball \#1; at time $T_{2}$, the robot draws the ball \#7 at random in the urn (containing the balls \#2 to \#n), replaces it in the urn and then expels the ball \#2; at time $\mathrm{T}_{3}$, the robot draws the ball \#9 at random in the urn (containing the balls \#3 to \#n), replaces it in the urn and then expels the ball \#3; at time $\mathrm{T}_{4}$, the robot draws the ball $\# 6$ at random in the urn (containing the balls \#4 to \#n), replaces it in the urn and then expels the ball \#4; at time $\mathrm{T}_{5}$, the robot draws the ball \#5 at random in the urn (containing the balls \#5 to \#n), replaces it in the urn, expels the ball \#5 and then stops. Let us term this variation the diachronic two-urn case ${ }^{++}$. The novelty in this variation with regard to the preceding experiment, is that the random selection is performed gradually and is only made effective when the number of the randomly drawn ball equals the number corresponding to the temporal position, i.e. when the ball $\# i$ is drawn at $\mathrm{T}_{\mathrm{i}}$. This contrasts with the synchronic version of the experiment, where the random selection is made definitively at time $T_{1}$. An instance of the diachronic two-urn case ${ }^{++}$, where the random selection is only made effective at time $\mathrm{T}_{5}$, is as follows:

\begin{tabular}{ccccccc}
\hline time & $\mathrm{T}_{0}$ & $\mathrm{~T}_{1}$ & $\mathrm{~T}_{2}$ & $\mathrm{~T}_{3}$ & $\mathrm{~T}_{4}$ & $\mathrm{~T}_{5}$ \\
\hline flipping & tails & & & & & \\
\hline range & & $1-10$ & $2-10$ & $3-10$ & $4-10$ & $5-10$ \\
\hline random \# & 4 & 7 & 9 & 6 & 5 \\
\hline expelled balls \# & & 1 & 2 & 3 & 4 & 5 \\
\hline
\end{tabular}

It should be noted that in the diachronic two-urn case ${ }^{++}$, the probability of drawing the ball $\# n$ at $\mathrm{T}_{\mathrm{n}}$ still equals $1 / n$. Let us denote by $\mathrm{E}$ the fact of drawing the ball $\# 5$ at $\mathrm{T}_{5}$. It follows that the probability of drawing the ball $\# 5$ at $T_{5}$ if the urn contains 10 balls is such that $\mathrm{P}(\mathrm{E})=9 / 10 \times 8 / 9 \times 7 / 8 \times 6 / 7 \times 1 / 6$ $=1 / 10$.

At this step, it should be pointed out that the incremental two-urn case (whether synchronic or diachronic) does not face the above-mentioned criticisms concerning the atemporal-temporal disanalogy between the human situation corresponding to DA and the original two-urn case. For it has been shown that the human situation corresponding to DA, being temporal, cannot be put in analogy with the two-urn case, which is atemporal. By contrast, the incremental two-urn case is a temporal experiment. Thus, the incremental two-urn case meets the above mentioned requirements concerning the analogy and can be put legitimately in analogy with the human situation corresponding to DA. In this context, we now face a variation of DA which can be stated explicitly as follows:

(11) in the incremental two-urn case, a Bayesian shift of the prior probability of $\mathrm{H}_{\text {few }}$ ensues

$$
\text { the situation corresponding to DA is analogous to the incremental two-urn case }
$$

$\therefore$ in the situation corresponding to DA, a Bayesian shift of the prior probability of $\mathrm{H}_{\text {few }}$ ensues

And this last variation is not vulnerable to the above objection. The analogy with the urn is now plainly plausible, since both situations are temporal.

At this point, it is also worth scrutinising the consequences of the incremental two-urn case (whether synchronic or diachronic) on the Eckhardt et al. analysis. For in the incremental two-urn case, the number of each ball expelled from the device is indexed on the rank of its expulsion. For example, you draw the ball \#60000000000. But you also know that the preceding ball was \#59999999999 and that the penultimate ball was \#59999999998, etc. However, this does not prevent you from reasoning in the same way as in the original two-urn case and from concluding to a Bayesian shift in favour of the $\mathrm{H}_{\text {few }}$ 
hypothesis. In this context, the incremental two-urn case has the following consequence: the fact of being time-indexed does not entail that the ball number is not randomly chosen. Contrast now with the central claim of the Eckhardt et al. analysis that the birth rank of each human is not randomly chosen, but rather indexed on the corresponding temporal position. Sowers in particular considers that the cause of DA is the time-indexation of the number corresponding to the birth rank. ${ }^{15}$ But what the incremental two-urn case and the corresponding analogy demonstrates, is that our birth rank can be time-indexed and nevertheless considered as random for DA purposes. And this point can be regarded as a significant objection to Sowers' analysis. This last remark leads to consider that the concrete analysis presented by Sowers does not prove however sufficient to provide a solution to DA. For the problem is revived when one considers the analogy between on the one hand, the human situation corresponding to DA and on the other hand, the incremental two-urn case. One can think that it is this latter analogy which constitutes truly the core of the DA-like reasoning. In this context, Sowers' conclusion according to which his analysis leads to the demise of DA appears far too strong. Echoing Eckhardt, he has certainly provided additional steps leading towards a resolution of DA and clarified significant points, but Sowers' analysis does not address veritably the strongest formulations of DA.

\section{Refining the Eckhardt et al. Analogy}

Let us consider, on the other hand, the analogy with the numbered ball dispenser, which is characteristic of the Eckhardt et al. line of thought. As mentioned above, Eckhardt describes the consecutive token dispenser, where the tokens are expelled from the urn at constant rates ('one per minute'). Sowers also describes an analogous experiment, where the balls are expelled from the urn and numbered accordingly, at the constant ${ }^{16}$ rate of one per minute. In this last experiment, the balls are numbered in the order of their expulsion from the urn.

However, the numbered ball dispenser can be criticised on the grounds that its protocol seems inaccurately defined. This inaccuracy concerns in particular the mechanism that expels a given ball \#n at $\mathrm{T}_{\mathrm{n}}$. What makes the device stops at $\mathrm{T}_{\mathrm{n}}$ after the ball $\# n$ has been expelled? The numbered ball dispenser seems to be designed for whatever way of choosing a given ball. So, could it be said, whatever mechanism allowing the choice of the ball \#n would be acceptable. But this won't do as a response, I think. For consider for example a deterministic situation, where the total number of balls in the urn is already settled before the experiment begins. And suppose that a device chooses a ball at random at $\mathrm{T}_{1}$ in the urn, say $\# 5$, and expels then accordingly the balls $\# 1$ at $\mathrm{T}_{1}, \# 2$ at $\mathrm{T}_{2}, \# 3$ at $\mathrm{T}_{3}, \# 4$ at $\mathrm{T}_{4}, \# 5$ at $\mathrm{T}_{5}$ and then stops. It appears then that the corresponding situation is fully isomorphic with the synchronic two-urn case ${ }^{++}$. Thus, at least on one particular interpretation, the numbered ball dispenser proves to be identical to the synchronic two-urn case ${ }^{++}$. But as we have seen, this latter experiment leads to a straightforward Bayesian shift in favour of the $\mathrm{H}_{\mathrm{few}}$ hypothesis, in contrast with the numbered ball dispenser which leaves the prior probabilities unchanged. Arguably, such interpretation of the numbered ball dispenser should be discarded, given that it is at the opposite of the Eckhardt et al. standpoint. But this shows that the protocol of the numbered ball dispenser stands in need of refinement and must be defined more accurately. This urges us to search another interpretation of the protocol of the numbered ball dispenser that fits more adequately with the spirit of the Eckhardt et al. line of thought.

Let us consider, second, another interpretation. Such interpretation arises from Sowers' description of the numbered ball dispenser. Sowers mentions in effect that the last ball is \#7 ('In parallel with the original scenario, suppose the last ball withdrawn is marked with a seven'). Now let us repeat the

\footnotetext{
${ }^{15}$ Cf. Sowers (2002, p. 40): 'My claim is that by assigning a rank to each person based on birth order, a time correlation is established in essentially the same way that the stopwatch process established a correlation with the balls.'.

${ }^{16}$ It could be pointed out that both Eckhardt's and Sowers' experiments do not exactly correspond to the human situation corresponding to DA. For in this latter situation, the humans appear on Earth at variable intervals of time, while Eckhardt and Sowers consider constant rates. However, this last disanalogy can be regarded as a minor qualm. For both Eckhardt's and Sowers' experiments could be eventually restated with items which are expelled at irregular rates instead of constant ones. In this context, a constant rate numbered ball dispenser can even be regarded as a useful simplification.
} 
experiment many times. In the long run, the numbered ball dispenser will always yield the ball \#7 (arguably, any small number would do the job). Under this interpretation, the repeatability of the experiment shows that the numbered ball dispenser has a bias towards \#7. Although it should be acknowledged that this biased numbered ball dispenser is also one possible interpretation of the numbered ball dispenser, I don't think neither that it fits adequately with what Sowers has in mind. For it seems that Sowers is concerned with a last ball expelled which is marked with whatever number (recall 'In general, the $n$th ball withdrawn you mark with the number $n$ '). For that reason, this second interpretation should also be rejected.

Let us consider then a third alternative interpretation. For it seems that an adequate interpretation of the numbered ball dispenser must do justice to Eckhardt' idea that it is impossible to make a random selection when there exists numerous unborn members in the reference class. Both previous interpretations of the numbered ball dispenser failed to incorporate adequately this latter idea. But consider now the following variation of the numbered ball dispenser:

The synchronic numbered ball dispenser An opaque device contains an urn that has 10 balls at $\mathrm{T}_{0}$, but will ultimately contain at $\mathrm{T}_{11}$ either 10 or 1000 numbered balls. The final number of balls in the urn will be determined by the flipping of a fair coin at $\mathrm{T}_{0}$. If heads, 990 numbered balls (\#11 to \#1000) will be added in the urn at $\mathrm{T}_{11}$. If tails, no balls will be added at $\mathrm{T}_{11}$. At time $\mathrm{T}_{1}$, you randomly draw the ball \#e from the urn and then replace it in the urn. Then a device expels at $\mathrm{T}_{1}$ the ball $\# 1$, at $\mathrm{T}_{2}$ the ball $\# 2 \ldots$, at $\mathrm{T}_{\mathrm{n}}$ the ball $\# n$. Now, according to the outcome of the random drawing performed at $\mathrm{T}_{1}$, the device stops at $\mathrm{T}_{\mathrm{e}}$ when the ball \#e is expelled. You formulate the $\mathrm{H}_{\text {few }}$ and $\mathrm{H}_{\text {many }}$ assumptions relating to the total number of balls in the urn at $\mathrm{T}_{11}$ with $\mathrm{P}\left(\mathrm{H}_{\text {few }}\right)=\mathrm{P}\left(\mathrm{H}_{\text {many }}\right)=0.5$. You know all the above and you get the ball $\# 5$ at $\mathrm{T}_{5}$ when the device stops. You conclude then that the prior probabilities remain unchanged.

An instance of the synchronic numbered ball dispenser is then as follows:

\begin{tabular}{ccccccc}
\hline time & $\mathrm{T}_{0}$ & $\mathrm{~T}_{1}$ & $\mathrm{~T}_{2}$ & $\mathrm{~T}_{3}$ & $\mathrm{~T}_{4}$ & $\mathrm{~T}_{5}$ \\
\hline flipping & tails & & & & & \\
\hline range & & $1-10$ & $2-10$ & $3-10$ & $4-10$ & $5-10$ \\
\hline random \# & 5 & & & & \\
\hline expelled balls \# & 1 & 2 & 3 & 4 & 5 \\
\hline
\end{tabular}

The novelty in this variation is that the urn contains 10 balls at the beginning but 990 other balls are eventually added later, at $\mathrm{T}_{11}$, depending on the outcome of a coin's toss. If the coin lands tails, nothing is done at $\mathrm{T}_{11}$ and the urn remains with only 10 balls, the ball being drawn continuously in the range $[1,10]$. If the coin lands heads, 990 balls are added in the urn at $T_{11}$. In this last case, the ball is drawn in the range $[1,10]$ until $T_{10}$, but from $T_{11}$ onwards, the ball is drawn in the range $[1,1000]$. The protocol of this experiment can be described more generally in the following terms: if the urn contains only 10 balls, a ball is drawn randomly in the range [1, 10]; but if the urn contains 1000 balls, a ball is drawn randomly in the range $[1,1000]$. Thus, the ball is drawn randomly, according to the actual number of balls in the urn. At this step, it should be apparent that this latter protocol does justice to Eckhardt's idea that it is impossible to make a random selection when there exists numerous unborn members in the reference class. In the present experiment the 990 balls that are added at $T_{11}$ represent those unborn members and the random process operates in the range $[1,10]$ until $\mathrm{T}_{10}$, even in the case where the reference class will ultimately contain 1000 balls. In this context, the synchronic numbered ball dispenser appears well as a robust variation of the numbered ball dispenser, which fully incorporates Eckhardt's insight. This latter variation does not face the above criticism of inaccuracy in its protocol. In this last situation, it would be plainly erroneous to conclude to a Bayesian shift in favour of the $\mathrm{H}_{\text {few }}$ hypothesis. What is rational to infer in this situation, though, is that the prior probabilities remain unchanged.

At this step, it is worth noting that the synchronic numbered ball dispenser has another virtue. In effect, it also incorporates an element of randomness, given that the number of the ball is first drawn randomly in $[1,10]$. Hence, the corresponding variation is much in line with the intuition that we are 
in some sense random humans. Consequently, this variation does justice to the idea that at least in some intuitive sense, our birth rank can be considered as random.

At this step, it is worth pointing out that a diachronic variation of the preceding experiment can even be envisaged. In particular, a diachronic numbered ball dispenser can be conceived of, where the random process is performed gradually instead of at a given time (i.e. at time $\mathrm{T}_{1}$ ) can be envisaged. It suffices then to replace the synchronic random drawing of the ball \#e of the preceding experiment, by a diachronic one, which runs as follows: at $\mathrm{T}_{\mathrm{i}}$, the robot draws a ball at random in the urn (now containing the balls $\# i$ to \#n) and the device expels the ball \#i; if the ball $\# i$ has been at $T_{i}$ then the device stops at $\mathrm{T}_{\mathrm{i}}$. An instance of the diachronic numbered ball dispenser is then as follows:

\begin{tabular}{ccccccc}
\hline time & $\mathrm{T}_{0}$ & $\mathrm{~T}_{1}$ & $\mathrm{~T}_{2}$ & $\mathrm{~T}_{3}$ & $\mathrm{~T}_{4}$ & $\mathrm{~T}_{5}$ \\
\hline flipping & tails & & & & & \\
\hline range & & $1-10$ & $2-10$ & $3-10$ & $4-10$ & $5-10$ \\
\hline random \# & 8 & 3 & 9 & 9 & 5 \\
\hline expelled balls \# & 1 & 2 & 3 & 4 & 5 \\
\hline
\end{tabular}

\section{The Third Route}

Given the above developments, we are now in a position to evaluate the adequacy of the analogy underlying DA. We now face two competing analogies with the human situation corresponding to DA. At this stage, the question that arises is the following: Is the human situation corresponding to DA analogous to (i) the two-urn case ${ }^{++}$or to (ii) the numbered ball dispenser? As we have seen, each model comes in two variations - i.e. synchronic or diachronic - which constitute strong variations of their respective original models, since they are not vulnerable to several objections that can be pressed against their original ancestors. At this step, the following question ensues: Does there exist an objective criterion allowing the preferential choice of one of the two competing models? Is there any clue that allows for preferring either the two-urn case ${ }^{++}$or the numbered ball dispenser to model the human situation corresponding to DA? Let us proceed now to review whether any such objective criterion is available.

Both Leslie and Eckhardt give reasons to justify the preferential choice of their favourite model. I shall examine these latter justifications in turn. To begin with, Leslie's motivation for the preferential choice of the two-urn case ${ }^{++}$results from countering a commonly raised objection based on the consideration that people of the future are not alive yet. The corresponding line of defence notably results from the emerald experiment (1996, p. 20): ${ }^{17}$

\footnotetext{
Imagine an experiment planned as follows. At some point in time, three humans would each be given an emerald. Several centuries afterwards, when a completely different set of humans was alive, five thousands humans would again each be given an emerald in the experiment. You have no knowledge, however, of whether your century is the earlier century in which just three people were to be in this situation, or the later century in which five thousand were to be in it. Do you say to yourself that if yours were the earlier century then the five thousand people wouldn't be alive yet, and that therefore you'd have no chance of being among them? On this basis, do you conclude that you might just as well bet that you lived in the earlier century?

Suppose you in fact betted that you lived there. If every emerald getter in the experiment betted in this way, there would be five thousand losers and only three winners. The sensible bet, therefore, is that yours is instead the later century of the two.
}

Leslie's remark is targeted at demonstrating (successfully, I think) that a DA-like reasoning can validly take place, even if there exist some unborn members in the reference class, whose coming into existence is already firmly planned at the time of the experiment. Nevertheless, Leslie acknowledges that DA is considerably weakened if our world is of an indeterministic nature, i.e. if the total number of humans who will ever have lived is not presently settled. Leslie acknowledges in effect that DA

\footnotetext{
${ }^{17}$ Cf. also Leslie (1993, p. 489).
} 
must be weakened if the fate of the human race is indeterministic when he evokes: ${ }^{18}$ '(...) the potentially much stronger objection that the number of names in the doomsday argument's imaginary urn, the number of all humans who will ever have lived, has not yet been firmly settled because the world is indeterministic'. At this step, it should be acknowledged that an indeterministic situation corresponds adequately to the numbered ball dispenser. Arguably, this latter model could be slightly modified to account for an indeterministic situation, notably by running the experiment with a quantum coin.

On the other hand, Eckhardt's justification for the preferential choice of the numbered ball dispenser is twofold. Eckhardt stresses, first, that our birth rank is non-random. But there is a strong intuition that we are, at least for a given reference class, random humans and consequently, our birth rank can also be random. But Eckhardt argues, second, and more convincingly, for the impossibility of drawing a random number without knowing the total size of the reference class. The different variations of the numbered ball dispenser described above incorporate this latter insight. However, one possible weakness in Eckhardt's account is that he denies the analogy with the lottery case (in our framework, the two-urn case $^{++}$) in all cases. But how can we have the certainty that an analogy with the two-urn case $^{++}$does not hold, for a given reference class? It appears that we lack the relevant evidence to rule out such a hypothesis with absolute certainty.

Although Leslie and Eckhardt offer strong justifications for their favourite model, it appears that both of them do not provide sufficient objective motivation for ruling out the opposite model. On the one hand, Leslie openly accepts that his favourite analogy with the lottery case could fail in some indeterministic circumstances, thus leaving room to some variation of the numbered ball dispenser to apply. On the other hand, Eckhardt does not expose the objective motivation for rejecting the analogy with the two-urn case ${ }^{++}$in all cases. The upshot is that we are still left with an indeterminate situation.

Let us then investigate whether any of the two competing models has an advantage over the other for modelling accurately the specific features of the human situation corresponding to DA. It is worth pointing out preliminarily that from an external viewpoint, there is no difference between the two models. In effect, from an observer's viewpoint, the external sequence consists in the expulsion of the balls \#1 at $\mathrm{T}_{1}, \# 2$ at $\mathrm{T}_{2}, \# 3$ at $\mathrm{T}_{3}, \ldots$, \#e at $\mathrm{T}_{\mathrm{e}}$, whether one considers the two-urn case ${ }^{++}$or the numbered ball dispenser. This corresponds adequately to the temporal feature of the human situation corresponding to DA. Hence, from this viewpoint, the analogy proves to be strongly established in both cases.

Let us turn now to the internal part of both experiments, concerning in particular what relates to the random process. As we have seen, both models admit of a synchronic variation where the random drawing is made at $T_{0}$ and a diachronic variation where the random drawing is made gradually from $T_{1}$ to $\mathrm{T}_{\mathrm{e}}$. In consequence, both models are capable of modelling adequately the random process ${ }^{19}$ that determines the birth rank of each human. Whether such random drawing has been made before the beginning of humankind or perhaps more plausibly, during the course of the existence of the human race doesn't matter. For the two-urn case ${ }^{++}$and the numbered ball dispenser both admit of respective variations which are capable of handling adequately these two types of situations. At this stage, it appears that both models are capable of modelling adequately the random process that determines the birth rank of each human. From this viewpoint, the two competing models are still on a par for the modelisation of situations which differ according to the synchronic/diachronic distinction.

To sum up now. From the above, it results that both models admit of respectively two variations, i.e. synchronic or diachronic. An immediate consequence is that the two models are equally suited for modelling adequately the different modalities of the human situation corresponding to DA, depending on the synchronic/diachronic distinction. The upshot is that this equally powerful modelling ability of the two competing models still leaves us with an indeterminate situation. At this stage, it appears that we lack an objective criterion allowing to decide rationally whether the two-urn case ${ }^{++}$or the numbered ball dispenser is the relevant model for the human situation corresponding to DA. In the lack of objective evidence, it is then wise to apply a principle of indifference, which leads to retain

\footnotetext{
${ }^{18}$ Cf. Leslie (1993, p. 490).

${ }^{19}$ To put it metaphorically. Needless to say, such random process need not be taken at face value.
} 
both models as roughly equiprobable. At this stage, it appears that we are on a third route: either the two-urn case ${ }^{++}$or the numbered ball dispenser applies to the human situation corresponding to DA. In this context, both Leslie's and Eckhardt's positions can be regarded as monist attitudes. For the preferential choice of either the two-urn case ${ }^{++}$or the numbered ball dispenser appears well as a onesided attitude. By contrast, the present view, based on a third route, is of a pluralist nature. For it appears that an adequate model should reflect the fundamental property of being two-sided. Perhaps if the present view were grounded on a purely disjunctive model, i.e. corresponding to the definition that either Leslie's or Eckhardt's position were true, there would be serious grounds for doubting that there would be something interestingly novel in the third route. But as will become clearer later, the third route allows for both two-urn case ${ }^{++}$and numbered ball dispenser to apply simultaneously to the human situation corresponding to DA. The essence of the third route is then pluralistically based on a conjunctive treatment of both Leslie's and Eckhardt's models. In short, both models apply in some cases. But explaining how both models apply without appealing to any paraconsistent logic requires that we delve more deeply into the underpinnings of the reference class problem.

\section{The Reference Class Problem}

Let us recall now the reference class problem..$^{20}$ Roughly, it is the problem of how to define 'humans'. More accurately, it can be stated as follows: How can the reference class be objectively defined for DA-purposes? For an extensive or restrictive definition of the reference class could be provided. An extensively defined reference class would include for example the somewhat exotic future evolutions of humankind, for example with an average I.Q. of 200 or with backward causation abilities. Conversely, a restrictively designed reference class would only include those humans who correspond accurately to the characteristics of, say, homo sapiens sapiens, thus excluding the past homo sapiens neandertalensis and the future homo sapiens supersapiens. To put it more in adequation with our current taxonomy, the reference class can be defined at different levels which correspond respectively to the supergenus superhomo, the homo genus, the homo sapiens species, the homo sapiens sapiens subspecies, etc. At this step, it appears that we lack an objective criterion to choose the corresponding level non-arbitrarily.

Leslie's treatment of the reference class problem is exposed in the response made to Eckhardt (1993) and in The End of the World (1996). ${ }^{21}$ Leslie's solution to the reference class problem goes as follows: One can choose the reference class more or less as one wishes, i.e. at whatever level of extension or of restriction. Once this choice is performed, it suffices to adjust the prior probabilities accordingly to get the argument moving. Leslie's sole condition is that the reference class should not be chosen at an extreme level of extension or of restriction. ${ }^{22}$ Furthermore, Leslie addresses the resulting fact that each human belongs to several different classes, restrictively or extensively defined. However, this is not a problem from Leslie's standpoint, since the argument works for all classes. In effect, a Bayesian shift ensues for whatever reference class arbitrarily chosen, at a somewhat reasonable level of extension or of restriction. Leslie illustrates this point with an urn analogy. By contrast with the one-colour two-urn case, he considers an urn that contains balls of different colours, say red and green. A red ball is drawn from the urn. From a restrictive viewpoint, the ball is a random red ball and there is no difference in this case with the classical two-urn case. But from a more extensive viewpoint, the ball is also a random red-or-green ball. ${ }^{23}$ According to Leslie, although the prior probabilities are different in each

\footnotetext{
${ }^{20}$ The reference class problem in probability theory is notably exposed in Hájek (2002, s. 3.3). For a treatment of the reference class problem in the context of DA, see notably Eckhardt (1993, 1997), Bostrom (1997, 2002, ch. 4 pp. 69-72 and ch. 5), Franceschi (1998, 1999). The point of Franceschi (1999) can be construed as a treatment of the reference class problem within confirmation theory.

${ }^{21}$ In the part entitled 'Just who should count as being human?' (pp. 256-63).

${ }^{22}$ Cf. 1996, p. 260: 'Widenings of reference class can easily be taken too far.' and p. 261: 'Again, some ways of narrowing a reference class might perhaps seem inappropriate'.

${ }^{23}$ Cf. Leslie (1996, p. 259): 'Suppose all the balls in the urn are numbered. A ball is drawn. It turns out to be bright red. Note that it is not only a bright red ball whose number has been drawn at random from the numbers of all the bright red balls in the urn, but also a red-or-reddish ball whose number has been drawn at random from the numbers of all the red-or-reddish balls in the urn'.
} 
case, a Bayesian shift ensues in both cases. ${ }^{24}$ In sum, on Leslie's view, the reference class problem can be overcome because the argument works for all somewhat reasonably defined reference classes.

By contrast, Eckhardt's treatment of the reference class problem stresses the difficulty, for the same individual, of being simultaneously random in several different classes: '(...) do we have better reason to believe that we are random human than random vertebrates or random social animals? Can the same item be random in all these classes? Isn't a random human a rather exceptional vertebrate?' (1993, p. 483). At this step, it should be acknowledged that an adequate treatment of the reference class problem should address that relevant objection. We shall then return to this reservation later.

At this stage, one might wonder what treatment of the reference class problem results from the third route. To begin with, let us reframe the reference class problem into the above-mentioned taxonomy of experiments. Now it appears that both two-urn case ${ }^{++}$and numbered ball dispenser can be easily adapted, in order to incorporate the elements of the reference class problem.

\subsection{The two-colour two-urn case ${ }^{++}$}

Let us begin with the two-urn case ${ }^{++}$. Consider then a two-colour experiment where the relevant class is the class of red-or-green balls, instead of the class of red balls. To fix ideas, consider that the first 10 balls (\#1 to \#10) are red and the other 990 balls (\#11 to \#1000) are green. We get then straightforwardly the following variation of the two-urn case $^{++}$, applied to red-or-green balls:

The two-colour two-urn case ${ }^{++}$An urn is in front of you, and you know that it contains, depending on the flipping at time $\mathrm{T}_{0}$ of a fair coin, either 10 (tails) or 1000 (heads) numbered red-or-green balls. At time $T_{1}$, you randomly draw the ball \#e from the urn. Then a device expels at $\mathrm{T}_{1}$ the ball \#1, at $\mathrm{T}_{2}$ the ball \#2..., and finally at $\mathrm{T}_{\mathrm{e}}$ the ball \#e. Once the ball \#e is expelled, the device stops. You formulate the $\mathrm{H}_{\text {few }}$ and $\mathrm{H}_{\text {many }}$ assumptions with $\mathrm{P}\left(\mathrm{H}_{\text {few }}\right)=$ $\mathrm{P}\left(\mathrm{H}_{\text {many }}\right)=0.5$ and you try to evaluate the number of red-or-green balls which were contained at $\mathrm{T}_{0}$ in the urn. You know all the above and you get the red ball \#5 at $\mathrm{T}_{5}$ when the device stops. You conclude then to an upward Bayesian shift in favour of the $\mathrm{H}_{\text {few }}$ hypothesis.

Within this variation, the urn is filled with red-or-green balls and a given red ball (and also whatever green ball) can also be considered as a red-or-green ball. At this step, it should be apparent that this variation of the two-urn case applied to the class of red-or-green balls yields a straightforward Bayesian shift, in the same way as the original variation applied to the class of red balls. It is also worth noting that the two-colour two-urn case ${ }^{++}$is fully in adequation with Leslie's treatment of the reference class problem. The key point is that the red ball which is randomly drawn from the urn can be legitimately considered as random in both the class of red-or-green balls and the class of red balls. In this particular case, this invalidates Eckhardt's objection that a given ball cannot be random in two different classes. In the same way, it should be pointed out that in this case, the third route's standpoint has also nothing novel to say.

\subsection{The two-colour numbered ball dispenser}

Let us turn now to the numbered ball dispenser. Consider then a two-colour experiment, where, just as previously, the first 10 balls (\#1 to \#10) are red and the other 990 balls (\#11 to \#1000) are green, in replacement of the original one-colour experiment. We get then accordingly the following two-colour variation of the numbered ball dispenser:

\footnotetext{
${ }^{24}$ Cf. Leslie (1996, pp. 258-9): 'The thing to note is that the red ball can be treated either just as a red ball or else as a red-or-green ball. Bayes's Rule applies in both cases. When we're interested in how many red balls there are in the urn, we need to treat the ball just as a red ball. The 'prior probabilities' entering into our Bayesian calculation are then probabilities for such and such numbers of red balls. When, in contrast, what interests us is how many red-or-green balls the urn contains, then we have to treat the red ball as red-or-green. Correspondingly, the prior probabilities entering into the calculation are the prior probabilities of various numbers of balls in the red-or-green-ball class. [...] All this evidently continues to apply to when being-red-orgreen is replaced by being-red-or-pink, or being-red-or-reddish'.
} 
The two-colour numbered ball dispenser An opaque device contains an urn that has 10 red balls at $\mathrm{T}_{0}$, but will ultimately contain either 10 red balls or 1000 red-or-green numbered balls, consisting of 10 red balls and 990 green balls. The final number of red-or-green balls in the urn will be determined by the flipping of a fair coin at $T_{0}$. If heads, 990 green numbered balls (\#11 to \#1000) will be added in the urn at $\mathrm{T}_{11}$. If tails, no green balls will be added at $\mathrm{T}_{11}$. At time $\mathrm{T}_{1}$, you randomly draw the ball \#e from the urn and then replace it in the urn. Then a device expels at $T_{1}$ the ball $\# 1$, at $T_{2}$ the ball $\# 2 \ldots$, at $T_{n}$ the ball $\# n$. Now, according to the outcome of the random drawing performed at $\mathrm{T}_{1}$, the device stops at $\mathrm{T}_{\mathrm{e}}$ when the ball \#e is expelled. You formulate the $\mathrm{H}_{\text {few }}$ and $\mathrm{H}_{\text {many }}$ assumptions relating to the total number of red-or-green balls in the urn at $\mathrm{T}_{11}$ with $\mathrm{P}\left(\mathrm{H}_{\text {few }}\right)=\mathrm{P}\left(\mathrm{H}_{\text {many }}\right)=0.5$. Now you know all the above and you get the red ball \#5 at $\mathrm{T}_{5}$ when the device stops. You conclude then that the prior probabilities remain unchanged.

Let us place ourselves, first, from the viewpoint of the extensively defined class of red-or-green balls. It appears then that this variation of the numbered ball dispenser is in all respects analogous with the one-colour variation. For considering the class of red-or-green balls instead of the class of red balls does not add anything new, since the structure of the original experiment is fully preserved. In this type of situation, it is then rational to conclude in the same way that in the original one-colour numbered ball dispenser and leave the prior probabilities unchanged.

But what if we focus, in the above experiment, only on the class of red balls? As a preliminary step, we need to adjust our hypotheses and the associated prior probabilities. We should ignore then whatever green ball drawn from the urn and focus only on red balls. Now drawing the red ball \#5 at random, we can legitimately conclude to a Bayesian shift in favour of the $\mathrm{H}_{\text {few }}$ hypothesis related to red balls. It is then patent that we reason just as in the two-urn case $^{++}$(whichever variation - whether synchronic or diachronic - will do the job). The upshot is that, from the restrictive viewpoint of the class of red balls, the situation is fully isomorphic with the one-colour two-urn case.

To sum up now. In the above situation, the numbered ball dispenser applies to the class of red-orgreen balls while the two-urn case applies to the more restrictive class of red balls. This casts light on the fact that a two-urn case concerning red balls is encapsulated in a numbered ball dispenser related to red-or-green balls. And according to the conjunctive essence of the third route, it appears that the two-urn case $e^{++}$holds from a restrictive viewpoint and the numbered ball dispenser applies from a more extensive standpoint. Now fully in accordance with the first route, it appears that both competing models apply to different reference classes. To put it another way: while the class of red balls disappears fast, the class of red-or-green balls survives later. From the third route's viewpoint, there exists one class for which Leslie's model works and one slightly more extensive other class for which Eckhardt's model prevails.

\subsection{The neandertalian case}

At this step, we are in a position to illustrate the above point through a concrete example related to our past situation. Leslie notably addresses the case of a neandertalian who would have implemented a DA-like reasoning: ${ }^{25}$

Consider the protest that any Stone Age man who had used the argument would have been led to the erroneous conclusion that the human race would soon die out. A first reply is: So what? It is not a defect in any merely probabilistic argument if it leads someone improbably situated - someone very early in time, maybe, or someone who has thrown a dozen dice with eyes shut and expects (mistakenly, in view of what is actually on the table) not to see a dozen sixes upon opening them - to an erroneous conclusion.

From the fact that Leslie considers the neandertalian's conclusion as erroneous, it is implicit here that Leslie assimilates the reference class to the somewhat extensively defined homo sapiens species (the conclusion would have been true, had the neandertalian considered the more restrictive class of the homo sapiens neandertalensis subspecies). Leslie's treatment of the neandertalian case is thus fully in

\footnotetext{
${ }^{25}$ Cf. (1992, pp. 527-8).
} 
adequation with the two-urn case $e^{++}$. Leslie acknowledges the fact that a neandertalian who would have implemented a DA-like reasoning related to the homo sapiens reference class would have been led to the false conclusion of a nearest extinction. But on Leslie's view, this is due to the above-mentioned ${ }^{26}$ fact that the reasoning based on the two-urn case does not yield absolute certainty. For it works in most cases, but exceptionally early members of the reference class can be led to an erroneous conclusion, despite the overall soundness of their DA-like reasoning.

Let us analyse now the neandertalian case from the third route's standpoint. I shall assume in the following that our current taxonomy is the best objective guide at our disposal for drawing relevant distinctions among reference classes. This has the effect of limiting the choice of the reference class to three main options: the subspecies homo sapiens neandertalensis, the species homo sapiens and the homo genus. Historically, the homo sapiens neandertalensis subspecies has appeared on the earth near $-200.000 \mathrm{BCE}$ and then became extinct near -35.000 BCE. On the other hand, our current subspecies homo sapiens sapiens has appeared near $-120.000 \mathrm{BCE}$. From the above, it results that, from the viewpoint of a neandertalian, we need to distinguish two cases: (i) from -200.000 to $-120.000 \mathrm{BCE}$, homo sapiens neandertalensis was the sole representative on the earth of the homo sapiens species. By contrast, from (ii) -120.000 to $-35.000 \mathrm{BCE}$, the earth was populated simultaneously with two coexistent members of the homo sapiens species, i.e. homo sapiens neandertalensis and homo sapiens sapiens.

Let us begin then (i) with the first period, i.e. from -200.000 to -120.000 BCE. At this time, homo sapiens neandertalensis is the unique representative on the earth of the homo sapiens species, but also of the homo genus, of the superhomo supergenus, etc. Now the question is: Which reference class is relevant for DA-purposes? The point is that the neandertalian can consider himself/herself at a somewhat restrictive level, as a member of the homo sapiens neandertalensis subspecies, or at a slightly more extensive level as a member of the homo sapiens species. ${ }^{27}$ Now it appears that there would have been nothing wrong had the neandertalian identified the reference class with homo sapiens neandertalensis. For the neandertalian had serious grounds for considering himself/herself as a random sample of the somewhat restrictively defined homo sapiens neandertalensis class. In this case, the neandertalian would have concluded, in virtue of the third route's line of reasoning, that either the two-urn case ${ }^{++}$or the numbered ball dispenser applies to the homo sapiens neandertalensis class.

On the other hand, had the neandertalian identified the reference class with the wider homo sapiens species, an immediate qualm would have pressed: Is a member of the homo sapiens neandertalensis class (red balls) representative of the wider homo sapiens (red-or-green balls) class? For this latter class, the reasoning of the neandertalian goes, could include further members such as, say, homo sapiens sapiens (green balls), who could well appear during the course of evolution. And the point is, the neandertalian could pursue, that I only see members of the homo sapiens neandertalensis subspecies around me, and no representative of other subspecies belonging to the homo sapiens species. This gives me strong grounds for not considering myself (and my associated birth rank) as a random sample of the homo sapiens class. To put it in terms of balls: since I only draw red balls, I cannot reasonably consider these latter as representative samples of the class of red-or-green balls, in order to draw inductive conclusions. Put otherwise: drawing only red balls gives me strong grounds for preferring the hypothesis that the urn contains only red balls over the hypothesis that it contains red and green balls. Now the fact that he/she cannot consider himself/herself as a random sample of the wider homo sapiens class, precludes the neandertalian from applying the DA-like reasoning to this latter class. The upshot is that the homo sapiens neandertalensis subspecies is the relevant class for DA-purposes in the neandertalian case at the period from -200.000 to -120.000 BCE.

Let us turn now (ii) to the second period from (ii) -120.000 to -35.000 BCE. At this time, there are simultaneously two representative subspecies of the homo sapiens species (red-or-green balls) which cohabit on the earth: homo sapiens neandertalensis (red balls) and homo sapiens sapiens (green balls). Now the neandertalian could choose, just as previously, the homo sapiens neandertalensis as an appropriate reference class and reason in a similar fashion. But in this case, the neandertalian could also legitimately choose the wider homo sapiens class. For the neandertalian had then strong grounds

\footnotetext{
${ }^{26}$ Cf. $\S 1$.

${ }^{27}$ To simplify matters, only two competing reference classes are envisaged here. The same goes if one extends the choice to other wider subdivisions of the taxonomy, such as the homo genus, the superhomo supergenus, etc.
} 
for considering that he/she is randomly drawn from the homo sapiens (red-or-green balls) class, since he/she observes both representatives of the homo sapiens neandertalensis (red balls) subspecies and of the homo sapiens sapiens subspecies (green balls). The upshot is that either the homo sapiens neandertalensis or the homo sapiens species is a convenient reference class for DA-purposes in the neandertalian case at the period from -120.000 to -35.000 BCE.

\subsection{Our present situation}

At this step, we are in a position to handle our present situation. Begin with the choice of the appropriate reference class. Will we consider the homo sapiens sapiens subspecies or the homo sapiens species as relevant for DA-purposes? At present time, it is patent that there is only one single representative subspecies - i.e. homo sapiens sapiens - of the homo sapiens species on the earth. Now for the same reasons that the neandertalian during the first period from -200.000 to -120.000 BCE, this precludes us from choosing the wider homo sapiens species as an appropriate reference class. This leaves us with a reference class which identifies itself with the homo sapiens sapiens subspecies.

At this stage, according to the third route, there exists equal grounds for applying either the two-urn case $^{++}$or the numbered ball dispenser to the reference class consisting of homo sapiens sapiens. Now applying a weak principle of indifference, we get a probability of $1 / 2$ that the two-urn case $e^{++}$holds and the same probability of $1 / 2$ that the numbered ball dispenser applies. On closer scrutiny, this is not far from Leslie's own position. Recall: DA is significantly weakened if our world is indeterministic. And Leslie's conception of an indeterministic world fits well with the sort of device that constitutes the numbered ball dispenser.

Let us suppose now that the two-urn case ${ }^{++}$holds for the homo sapiens sapiens subspecies. Two cases need then to be distinguished. On the one hand, it could well be the case that the extinction of the homo sapiens sapiens subspecies will be followed by the appearance of the much-evolved homo sapiens supersapiens subspecies. For such type of situation is very common among evolutionary species. Finally, this would render DA innocuous, by depriving it of its initially associated terror. At the same time, this leaves room for the argument to be successful for a given reference class, but without its frightening and counterintuitive consequences. To put it in terms of the third route: the restricted homo sapiens sapiens class goes extinct while the more extended homo sapiens class survives. In this type of situation, the two-urn case ${ }^{++}$applies to the restricted homo sapiens sapiens class while the (two-colour) numbered ball dispenser prevails for the wider homo sapiens class. But this ambivalent feature has the effect of depriving the original argument from its initial terror. Finally, this gives a way of accepting the conclusion of DA by rendering the argument less counterintuitive than in its original formulation.

On the other hand, it could also be the case that the extinction of the homo sapiens sapiens subspecies will not be followed by the appearance of any slightly different species or subspecies having evolved from our current human race. In this type of situation, both the restricted homo sapiens sapiens and the more extended homo sapiens classes would go extinct simultaneously. It should be acknowledged that this sort of extinction of homo sapiens sapiens would correspond to a genuinely frightening Doomsday.

Finally, this leads finally to a novel formulation of the argument. What results from the foregoing developments is that the appropriate reference class for our present DA-purposes is the homo sapiens sapiens subspecies. In addition, the Doomsday Argument must be weakened in two ways. It should be acknowledged first that either the two-urn case $e^{++}$or the numbered ball dispenser applies to the homo sapiens sapiens reference class. A weak principle of indifference yields a proportion of $1 / 2$ for both cases. Moreover, a second weakening of the argument arises from the reference class problem. Let us place ourselves in the case where the two-urn case $^{++}$holds for the reference class consisting of homo sapiens sapiens. Now as we have seen, we need then to distinguish between two main situations: either this two-urn case ${ }^{++}$is embedded into a two-colour variation of the numbered ball dispenser (innocuous Doomsday), or not (frightening Doomsday). At this step, a second application of a weak principle of indifference yields a proportion of $1 / 2$ for these two cases. Given these two side-steps, the resulting novel formulation of the argument could well be more consensual than the original one.

To sum up now. Four cases need to be distinguished: 
(i) homo sapiens sapiens goes extinct fast and is not followed by the appearance of a slightly different subspecies (a two-urn case $^{++}$for homo sapiens sapiens and a two-urn case $^{++}$for homo sapiens, i.e. a monovalent two-urn case ${ }^{++}$)

(ii) homo sapiens sapiens goes extinct fast and is followed by the appearance of a slightly different subspecies (a two-urn case ${ }^{++}$for homo sapiens sapiens and a (two-colour) numbered ball dispenser for homo sapiens, i.e. an ambivalent two-urn case $^{++}$and also an ambivalent numbered ball dispenser)

(iii) homo sapiens sapiens goes extinct late and is not followed by the appearance of a slightly different subspecies (a (one-colour) numbered ball dispenser for homo sapiens sapiens and a (one-colour) numbered ball dispenser for homo sapiens, i.e. a monovalent numbered ball dispenser)

(iv) homo sapiens sapiens goes extinct late and is followed by the appearance of a slightly different subspecies (a (one-colour) numbered ball dispenser for homo sapiens sapiens and a (twocolour) numbered ball dispenser for homo sapiens, i.e. a monovalent numbered ball dispenser)

Now these four scenarios can be classified by increasing order of reassuring degree. We get then accordingly the following order: (i), (iii), (ii), (iv); the scenario (i) being the more frightening and the scenario (iv) the more reassuring. Hopefully, at this step, the resulting form of the Doomsday argument can be reconciled with our pretheoretical intuitions. For most scenarios, i.e. (ii), (iii) and (iv) are somewhat reassuring ones. Of course, we have not made fully disappear an impending frightening Doomsday, i.e. scenario (i). At this step, the moral is: accept (some limited) risk. However, don't give up your pretheoretical intuitions. In effect, taking into account the Doomsday argument now gives a proportion of $1 / 4$ for this frightening Doomsday scenario (based on a monovalent two-urn case $^{++}$) and a proportion of $3 / 4$ for other reassuring scenarios (based either on a monovalent or ambivalent numbered ball dispenser).

Lastly, what precedes casts light on an essential facet of the Doomsday Argument. For on a narrow sense, it is an argument about the fate of humankind. But on a broad sense (the one we have been concerned with) it emphasises the difficulty of applying probabilistic models to real-life situations, ${ }^{28}$ a difficulty which is usually largely underestimated. This opens a path to a whole field of practical interest, consisting of a taxonomy of probabilistic models, whose philosophical importance would have been unravelled without John Leslie's robust and courageous defence of the argument. ${ }^{29}$

\section{References}

Bostrom, N. (1997) 'Investigations into the Doomsday argument', preprint at http://www.anthropicprinciple.com/preprints/inv/investigations.html

Bostrom, N. (2002) Anthropic Bias: Observation Selection Effects in Science and Philosophy, New York, Routledge

Chambers, T. (2001) 'Do Doomsday's Proponents Think We Were Born Yesterday?', Philosophy, 76, 443-50

Delahaye, J-P. (1996) 'Recherche de modèles pour l'argument de l'apocalypse de Carter-Leslie', unpublished manuscript

Eckhardt, W. (1993) 'Probability Theory and the Doomsday Argument', Mind, 102, 483-88

Eckhardt, W. (1997) 'A Shooting-Room view of Doomsday', Journal of Philosophy, 94, 244-259

Franceschi, P. (1998) 'Une solution pour l'argument de l'apocalypse', Canadian Journal of Philosophy, 28, 227-46

\footnotetext{
${ }^{28}$ This important underpinning of the argument is also underlined in Delahaye (1996). This is also the main point of Sober (2003).

${ }^{29}$ I am grateful to Claude Panaccio and Daniel Andler for comments on an ancestor version of this paper. I especially thank Jean-Paul Delahaye for very useful comments and discussion. I am also indebted to John Leslie and Elliott Sober for comments on earlier drafts. I thank Nick Bostrom for very helpful discussion on the reference class problem.
} 
Franceschi, P. (1999) 'Comment l'urne de Carter et Leslie se déverse dans celle de Hempel', Canadian Journal of Philosophy, 29, 139-56, English translation under the title 'The Doomsday Argument and Hempel's Problem', PhilSci: 601

Franceschi, P. (2002) Une application des n-univers à l'argument de l'Apocalypse et au paradoxe de Goodman, Corté: University of Corsica, doctoral dissertation

Hájek, A. (2002) 'Interpretations of Probability', The Stanford Encyclopedia of Philosophy, E. N. Zalta (ed.), http://plato.stanford.edu/archives/win2002/entries/probability-interpret

Korb, K. \& Oliver, J. (1998) 'A Refutation of the Doomsday Argument', Mind, 107, 403-10

Leslie, J. (1992) 'Time and the Anthropic Principle', Mind, 101, 521-40

Leslie, J. (1993) 'Doom and Probabilities', Mind, 102, 489-91

Leslie, J. (1996) The End of the World: the science and ethics of human extinction, London, Routledge

Sober, E. (2003) 'An Empirical Critique of Two Versions of the Doomsday Argument - Gott's Line and Leslie's Wedge', Synthese, 135-3, 415-30

Sowers, G. F. (2002) 'The Demise of the Doomsday Argument', Mind, 111, 37-45 